\title{
ANALYSIS OF COVID-19 IMPACT ON NATURAL GAS SUPPLY RELIABILITY
}

\author{
Yichen $\mathrm{Li}^{1}$, Weichao Yu${ }^{1}$, Zihui Han ${ }^{2}$, Shuang Shi ${ }^{3}$, Weihe Huang ${ }^{4}$, \\ Kai Wen ${ }^{1}$, and Jing Gong ${ }^{*}$
}

In this study, we have analyzed the impact of COVID-19 on natural gas supply reliability. Natural gas supply reliability is defined as the ability to satisfy the market demand and is determined by both supply-side and demand-side policy. To evaluate the gas supply reliability of the natural gas pipeline system, we have applied the method of gas supply capacity calculation based on the results of the previous gas supply reliability studies. The method combines the unsteady flow hydraulic analysis, simulation of the state transition process, and the forecasting analysis of the demand and consumption. The analysis presents a case study based on the gas pipeline system in China. The analysis results indicate that the COVID-19 consequences will cause a decrease in gas supply reliability.

Keywords: COVID-19, gas supply reliability, demand side, market demand

\section{INTRODUCTION}

People around the world have to face great challenges brought by the coronavirus disease 2019 (COVID-19), which has not been experienced since the time of Spanish flu after the First World War [1]. China was the first country to implement the lockdown policy [2]. The policy led to a significant impact on the economy, environment, and energy supply chain [3-5]. The natural gas pipeline system is usually regarded

${ }^{1}$ National Engineering Laboratory for Pipeline Safety, China University of Petroleum, Beijing, China. ${ }^{2}$ Beijing Oil \& Gas Pipeline Control Center, Beijing 10010, China. ${ }^{3}$ Petrochina West East Gas Pipeline. ${ }^{4}$ China National Petroleum Corporation Beijing, China. Corresponding author: Jing Gong. E-mail: ydgj@cup.edu.cn Translated from Khimiya i Tekhnologiya Toplivi Masel, No. 6, pp. 91 - 94, November - December, 2020. 
as the main artery of the national energy system. The natural gas pipeline system is designed for stable and sustainable satisfaction of the market demand. In the previous studies, the gas supply reliability is estimated by the evaluation index, which defines the ability of the natural gas pipeline system to satisfy the market demand [6]. The gas supply reliability is determined by both supply-side and demand-side policies [7], while the supply side is defined by the gas supply capacity. In our previous studies [6-7], we have developed a method for the hydraulic analysis of unsteady flow based on state transition process simulation of components of the pipeline system and applied it to calculate the gas supply capacity. Since the natural gas pipeline system is little affected by COVID-19, we have applied the developed method to evaluate the gas supply capacity.

\section{METHODOLOGY}

The gas supply reliability index $R_{\text {system }}$ is defined to measure whether the natural gas pipeline system fulfills its responsibility and it can be expressed as follows:

$$
R_{\text {system }}= \begin{cases}1 & X \geq D \\ \frac{X}{D} & X<D\end{cases}
$$

where $X$ is the total amount of the natural gas supplied by pipeline system over the mission time, and $D$ is the total demand over the mission time.

\subsection{Gas supply capacity calculation}

The natural gas system consists of numerous components. If some of the components suffer from random failures or maintenance activities, it will affect the operating state of the whole system. As was shown in our previous studies, the first step of the gas supply capacity calculation is to simulate the state transition process. Simulation is based on creating the matrix of probabilities of transition between different states. The stochastic model of behavior is derived from the transition rate of each component, including failure rate, degrade rate, and repair rate. As a result of the simulation, the probability and duration of each state can be obtained. The second step of gas supply capacity calculation is the hydraulic analysis of unsteady flow. The hydraulic analysis is performed using the commercial software SPS (Stoner Pipeline Simulator), designed to consider the line pack effects. Finally, the simulation of all possible operating states is performed by using a

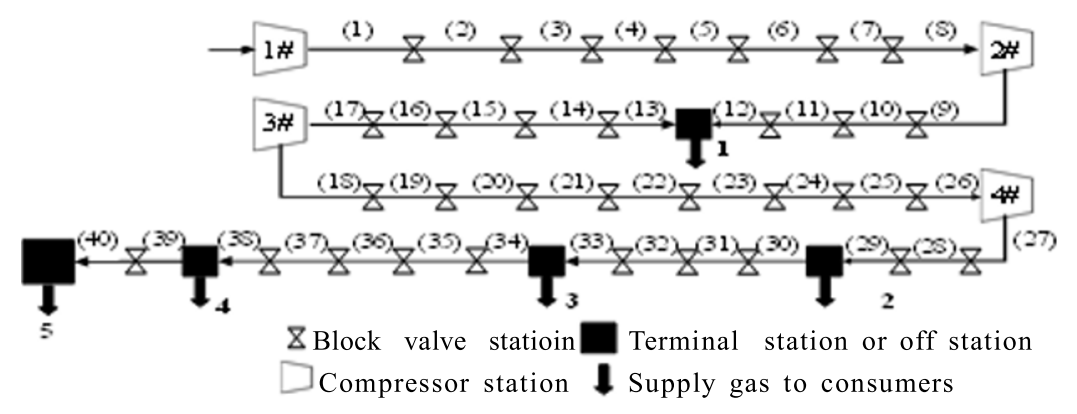

Fig. 1. Schematic diagram of the pipeline system. 
Monte Carlo-approach-based state transition process, and the amount of gas supplied is obtained by combining it with the hydraulic analysis.

\subsection{Demand Side}

In this paper, we consider the demand forecasting for the daily horizon. As mentioned before, there are numerous methods applicable to users with different characteristics. The first step is to establish the indicator to divide all users into groups, depending on which method is applied for forecasting. The fluctuation indicator $\lambda$ is established as follow:

$$
\lambda(i)=\frac{q_{\max }(i)-q_{\min }(i)}{q(i)}
$$

where $\lambda(i)$ is the fluctuation indicator of $i$ user in the evaluation period, $q_{\max }(i)$ is the maximum consumption, $q_{\text {min }}(i)$ is the minimum consumption, and $q(i)$ is the average consumption.

\section{CASE STUDY}

3.1 Background of the pipeline system in case study

In this paper, we consider a pipeline system in China for the case study analysis of the COVID-19 impact on natural gas supply reliability. The pipeline background is the same as was described in our previous study due to the limited impact of COVID-19 on the supply side. The case study pipeline system is an actual natural gas pipeline system in China operated by PetroChina. It has a total length of $929.6 \mathrm{~km}$ and

Table 1

\begin{tabular}{|c|c|}
\hline Node & $X_{\text {task }}\left(\mathrm{Nm}^{3} /\right.$ day $)$ \\
\hline 1 & 147941 \\
2 & 926030 \\
3 & 536700 \\
4 & 615100 \\
5 & 7900000 \\
\hline
\end{tabular}

Table 2

\begin{tabular}{|l|c|c|c|c|c|}
\hline \multicolumn{1}{|c|}{ User type } & Node 1 & Node 2 & Node 3 & Node 4 & Node 5 \\
\hline Urban gas distributor & $1.3 \%$ & $0.9 \%$ & $1.9 \%$ & $1.8 \%$ & $1.4 \%$ \\
CNG station & $-5.1 \%$ & $-4.1 \%$ & $-5.6 \%$ & $-3.8 \%$ & $-6.5 \%$ \\
Power plant & $1.4 \%$ & $1.7 \%$ & $0.2 \%$ & $0.4 \%$ & $1.4 \%$ \\
Industrial & $-9 \%$ & $-10.23 \%$ & $-9.3 \%$ & $-10.6 \%$ & $-12.9 \%$ \\
\hline
\end{tabular}



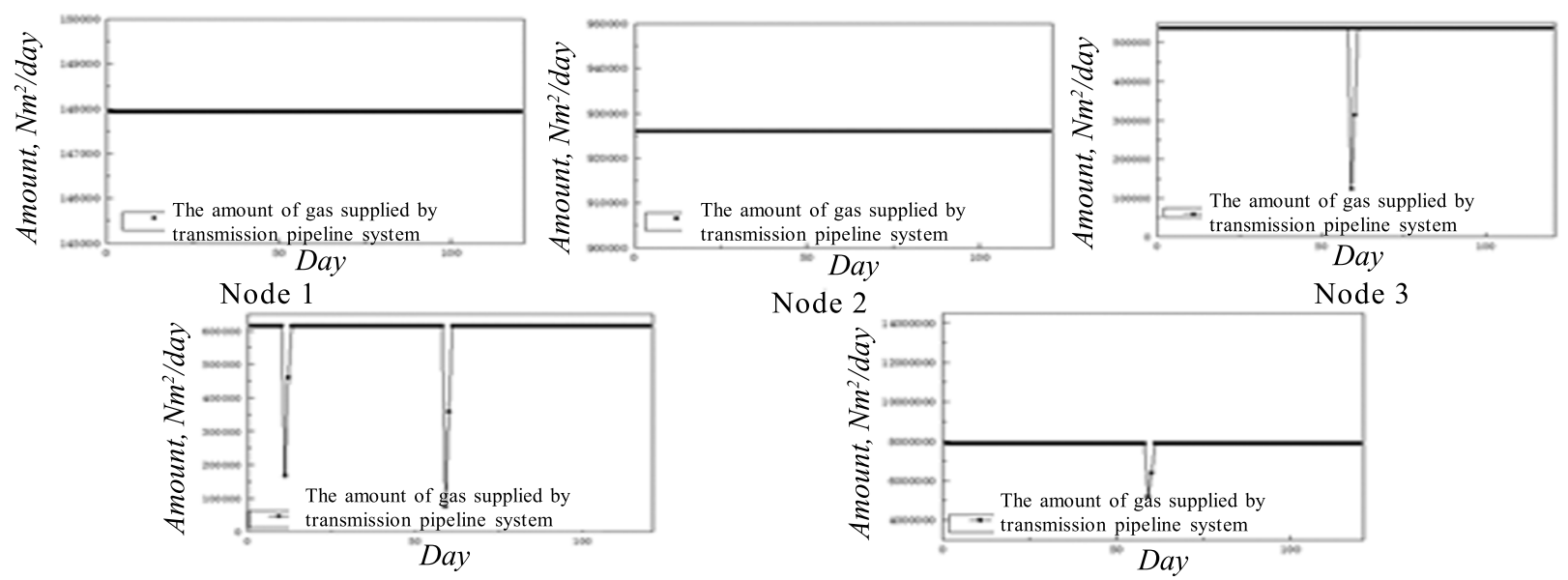

Node 4

Node 2 Node 3

Fig. 2. The result of gas supply capacity in one Monte Carlo trial.

includes 32 block valve stations, four offtake stations, one terminal station, and four compressor stations. The diameter of the pipeline is $660 \mathrm{~mm}$, the thickness is $7 \mathrm{~mm}$, and the inlet absolute pressure is $4.5 \mathrm{MPa}$. Figure 1 shows a schematic diagram of the system.

\subsection{Gas supply capacity calculation}

In gas supply capacity calculation, the pipeline segments are operating in one of two states: a good as new state and a failed state. The failure rate is $1.46 \times 10^{-8}(1 / \mathrm{h})$, and the repair rate is $0.0139(1 / \mathrm{h})$. The compressor stations consist of two running compressor units and one standby unit. The failure rate of each unit is $7.710^{-4}(1 / \mathrm{h})$, and the repair rate is assumed to be $0.025(1 / \mathrm{h})$. Table 1 shows the daily supply task. The duration of time in the case study is 120 days in winter, which corresponds to the highest demand in four seasons. The result of gas supply capacity in one Monte Carlo trial is shown in Fig.2.

\subsection{Demand side process}

As mention before, the COVID-19 influenced the natural gas consumption in the first half of the year, and the extent of the influence is shown in Table 2. To select the appropriate forecasting model, we have analyzed the consumption data of all users for the period from 01.01.2015 to 30.06.2020 and determined the fluctuation indicator for each user. First, we evaluate the forecasted demand for the period from 01.01.2020 to 30.06.2020. Then, applying the appropriate models to the consumption data for the period from 2015 to 2019, we evaluate the forcasted demand without considering the COVID-19 influence for the second half of the year. The demand of each user for the rest of the year is obtained by the following equation:

$$
d_{r}=d_{f} *(1-r)
$$

where $d_{r}$ is the user's forecasting demand for the rest of year 2020, $d_{f}$ is the forecasting demand without the COVID-19 influence, and $r$ is the difference ratio (as in Table 2). To obtain the demand of each user for the first quarter of 2021, the forecasting models are applied to the data for the period from 2015 to 2019 , and the 
Table 3

\begin{tabular}{|c|c|}
\hline Node & Daily average \\
\hline 1 & 0.999323 \\
2 & 0.999458 \\
3 & 0.994154 \\
4 & 0.982714 \\
5 & 0.97186 \\
\hline
\end{tabular}

Table 4

\begin{tabular}{|c|c|}
\hline Node & Daily average \\
\hline 1 & 0.99131 \\
2 & 0.98513 \\
3 & 0.98984 \\
4 & 0.97219 \\
5 & 0.95382 \\
\hline
\end{tabular}

results are combined with the forecasting demand without the COVID-19 influence. In conclusion, we calculate the forecasted demand of each user for the period from 01.11 .202 to 28.02.2021.

\subsection{Gas supply reliability assessment result}

Combining the results of the gas supply capacity and the forecasted demand, the gas supply reliability is calculated by Eq. (1). Table 3 shows the results without considering the COVID-19 influence. The results considering the COVID-19 influence are shown in Table 4.

\section{CONCLUSION}

This study applies three forecasting models suitable for different users with consumption background analysis. The case study indicates that the demand for the rest of the year may increase by $3 \%-4 \%$, and the demand in the first quarter of 2021 will increase by the normal growth rate, as before the COVID-19. The gas supply reliability evaluation shows that the COVID-19 has a negative influence on the gas supply reliability.

\section{REFERENCES}

1. J. J. Klemes, Y. V. Fan, and P. Jiang, "The energy and environmental footprints of COVID-19 fighting measures - PPE, disinfection, supply chains," Energy, 211, 118701 (2020).

2. J. Griffiths and A. Woodyatt, "780 million people in China are living under travel restrictions due to the coronavirus outbreak," https://www.cnn.com/2020/02/16/asia/coronavirus-covid-19-death-toll-update-intl-hnk/index.html (2020).

3. B. Rugani and D. Caro, "Impact of COVID-19 outbreak measures of lockdown on the Italian carbon footprint," Sci. Total Environ., 737, 139806 (2020).

4. D. Eryilmaz, M. Patria, and C. Heilbrun, "Assessment of the COVID-19 pandemic effect on regional electricity generation mix in NYISO, MISO, and PJM markets," Electricity J., 33(7), 106829 (2020). 
5. A. Naderipour, Z. Abdul-Malek, N. A. Ahmad, H. Kamyab, V. Ashokkumar, C. Ngamcharussrivichai, and S. Chelliapan, "Effect of COVID-19 virus on reducing GHG emission and increasing energy generated by renewable energy sources: A brief study in Malaysian context," Environ. Technol. Innov., 20, 101151 (2020).

6. W. Yu, S. Song, Y. Li, Y. Min, W. Huang, and K. Wen, "Gas supply reliability assessment of natural gas transmission pipeline systems," Energy, 162, 853-870 (2018).

7. W. Yu, J. Gong, S. Song, W. Huang, Y. Li, and J. Zhang, "Gas supply reliability analysis of a natural gas pipeline system considering the effects of underground gas storages," Appl. Energ., 252, 113458 (2019). 